\title{
Tempo de protrombina como marcador precoce de mortalidade em pacientes admitidos à Unidade de Terapia Intensiva
}

\author{
Prothrombin time as an early marker of mortality in patients admitted to the \\ intensive care unit
}

\author{
Nayana Camurça de Araújo', Benilton Batista de Souza², Arnaldo Aires Peixoto Junior ${ }^{3}$ \\ ${ }^{1}$ Acadêmica de Medicina da UNICID; ${ }^{2}$ Especialista em Clínica Médica pela Sociedade Brasileira de Clínica Médica; \\ ${ }^{3}$ Doutorando em Farmacologia pela Universidade Federal do Ceará (UFC)
}

\section{Resumo:}

Introdução: $O$ tempo de protrombina é um marcador da via extrínseca da coagulação, podendo refletir alterações na microcirculação. $\mathrm{O}$ objetivo deste estudo foi estabelecer uma associação entre o tempo de protrombina à admissão dos pacientes internados em Unidade de Terapia Intensiva (UTI) e seu prognóstico. Metodologia: Estudo retrospectivo com análise de 278 pacientes admitidos consecutivamente na UTI, durante o período de 01 de janeiro de 2010 a 31 de janeiro de 2011. Resultados: Houve discreto predomínio do sexo feminino, com idade média de $56,9 \pm 20,5$ anos. 0 tempo médio de permanência foi 7,9 $\pm 7,3$ dias e o APACHE II médio foi de $21,9 \pm 9,0$ pontos. Os pacientes com mortalidade precoce (primeiras $48 \mathrm{~h}$ de UTI) tiveram maior valor do tempo de protrombina, expresso por meio do INR $(2,48 \pm 1,9)$ que os sobreviventes $(1,4 \pm 0,5) ; p<0,05$. Conclusões: 0 tempo de protrombina se revelou, significativamente, maior entre os pacientes que faleceram precocemente, sugerindo ser um marcador prognóstico.

Palavras-chave: Tempo de Protrombina. Terapia Intensiva. Prognóstico. Mortalidade.

\begin{abstract}
:
Background: The prothrombin time is a marker of extrinsic pathway of coagulation, which can reflect changes in the microcirculation. The aim of this study was to establish an association between the prothrombin time on admission of patients in the intensive care unit (ICU) and its prognosis. Methodology: A retrospective study with analysis of 278 patients consecutively admitted to the ICU during the period from 01 January, 2010 to January 31, 2011. Results: There was a slight predominance of females with a mean age of $56.9 \pm 20.5$ years. The average length of stay was $7.9 \pm 7.3$ days and mean APACHE II score was $21.9 \pm 9$ points. Patients with early mortality (less than $48 \mathrm{~h}$ in ICU) had higher value of prothrombin time, expressed through the INR (2.48 \pm 1.9$)$ than survivors $(1.4 \pm 0.5), \quad p<0,05$. Conclusions: The prothrombin time proved to be significantly higher among patients who died early, suggesting a prognostic marker.

Keywords: Prothrombin Time. Intensive Care. Prognostic. Mortality.
\end{abstract}

\section{INTRODUÇÃO}

Um dos princípios básicos em Medicina Intensiva é tentar identificar disfunções em uma fase inicial com o objetivo de adotar as medidas de suporte apropriadas. Partindo desse princípio, tem-se lançado mão de escores prognósticos no intuito de se discriminar precocemente os pacientes com potencial para evoluírem de forma desfavorável durante a permanência na UTI.

Nesse sentido, há uma busca por marcadores que apresentem sensibilidade acurada para determinação de prognóstico, aliados a relação custo-benefício adequada e com fácil acesso para obtenção de resultado. Em meio às alternativas disponíveis, estudos demonstram a utilidade de monitorização dos níveis de lactato, saturação

Correspondência / Correspondence: Arnaldo Aires Peixoto Junior. Universidade Federal do Ceará, Centro de Ciências da Saúde, Hosp. Univ.Walter Cantidio. R.Capitão Francisco Pedro, 1290. Rodolfo Teófilo. 60000-000 - Fortaleza, CE - Brasil. Tel (85) 33668382. arnaldoaires@ hotmail.com venosa mista e déficit de bases., 2, 3, 4 No contexto da procura constante por um marcador mais acurado, de baixo custo e de fácil disponibilidade, outras alternativas vem sendo investigadas.

Nos últimos anos, estudos abordando a teoria do efeito das alterações da microcirculação na sepse trouxeram uma nova perspectiva quanto a esse assunto. ${ }^{5}$ Já está bem estabelecida a relação entre coagulação e inflamação, e com isso o desenvolvimento de novas estratégias terapêuticas objetivando interferir nas vias fisiopatológicas envolvidas, como o uso da Proteína $\mathrm{C}$ ativada recombinante ou Anti-trombina III. ${ }^{6}$ Também tem sido estudada a influência da coagulação como fator prognóstico independente, bem como sua capacidade de predizer evolução desfavorável de outros sistemas, ${ }^{7}$ mas ainda não se encontrou um marcador de fácil disponibilidade para avaliar as alterações da microcirculação causadas pelos estados de coagulopatia. Na literatura há relatos da associação positiva entre o valor da Relação 
Normatizada Internacional (INR) e os quadros de Coagulação Intravascular Disseminada (CIVD), sugerindo que as alterações na microcirculação poderiam estar relacionadas a alterações nas vias de coagulação. Também tem sido demonstrado o valor prognóstico do INR na avaliação de pacientes em doença hepática terminal, sendo inclusive o marcador de maior peso utilizado no sistema MELD para listagem para transplante. ${ }^{8,9}$

Considerando que o INR reflete uma das vias de coagulação do organismo e que as alterações da microcirculação parecem estar associadas com as alterações de coagulabilidade vistas nos quadros de sepse, parece haver uma correlação entre o INR e as alterações na microcirculação. Assim, o objetivo deste estudo foi avaliar a relação entre o INR e gravidade, o tempo de perma $n$ nência e a mortalidade dos pacientes admitidos na UTI.

\section{MATERIAIS E MÉTODOS}

O estudo foi realizado na Unidade de Terapia Intensiva (UTI) do Hospital Universitário Walter Cantídio da Universidade Federal do Ceará, composta de seis leitos destinados a pacientes adultos clínicos ou cirúrgicos. Trata-se de um estudo retrospectivo, com análise dos exames laboratoriais e prontuários de 278 pacientes interna-dos de forma consecutiva durante o período de 01 de janeiro de 2010 a 31 de janeiro de 2011. A gravidade dos pacientes foi inferida usando o APACHE II (escore e mortalidade prevista). O lactato arterial, o excesso de base e o tempo de protrombina (TAP), em segundos e em atividade percentual, assim como o INR, derivaram da análise da primeira amostra sanguínea colhida após a admissão na UTI para avaliação laboratorial de rotina. Buscouse identificar eventual correlação entre os valores de APACHE II, tempo de permanência na UTI e as taxas de mortalidade global, precoce $(<48 \mathrm{~h}$ ) ou tardia (> 48h) com as magnitudes do TAP/INR. O programa GraphPad for windows foi usado para realizar a análise estatística. Média e desvio-padrão foram calculados para as variáveis contínuas com distribuição normal. O teste do Qui-quadrado foi utilizado para avaliar variáveis qualitativas e categoriais, enquanto a Análise de Variância (ANOVA) foi usada para comparar médias de variáveis contínuas. Nos testes estatísticos foi calculado o valor do $\mathrm{p}$ e adotado o nível significativo de $5 \%$.

O estudo está de acordo com a resolução 196/96 do Conselho Nacional de Saúde e o projeto que o originou foi aprovado pelo Comitê de Ética em Pesquisa da Universidade Federal do Ceará.

\section{RESULTADOS}

Observou-se discreto predomínio do sexo feminino $(51,07 \%)$, com idade mediana de 59 anos (variação: 15-82 anos). O APACHE II médio foi de 21,9 $\pm 9,0$ pontos, com uma mortalidade prevista de $42,5 \%$. O tempo médio de permanência na UTI foi de $7,9 \pm$ 7,3 dias. A mortalidade geral dos pacientes durante a internação na UTI foi de $42,4 \%$ (precoce: $13,3 \%$; tardia: $29,1 \%)$.

Os valores do lactato arterial, excesso de bases, tempo de protrombina (em segundos e em porcentagem) e o INR foram comparados entre os pacientes que receberam alta da UTI e os pacientes que evoluíram a óbito (precoce e tardio) durante a estadia na UTI. Observou-se diferença do ponto de vista estatístico, através da análise de variância, nos valores do APACHE II, TAP (em segundos e em porcentagem), INR e excesso de bases entre os pacientes que receberam alta e os pacientes que evoluíram a óbito em menos de 48 horas e em mais de 48 horas (Tabela 1).

Tabela 1. Comparacão dos dados dos pacientes admitidos na UTI.

\begin{tabular}{lllll}
\hline \multicolumn{4}{l}{ Tabela 1: Comparação dos dados dos pacientes admitidos na UTI } & \\
\hline & Alta & Óbito $<48 \mathrm{~h}$ & Óbito $>48 \mathrm{~h}$ & $P$ \\
Idade* & $56(34-72)$ & $60(41,5-72,2)$ & $66(52,5-72,2)$ & $>0,05$ \\
APACHE II* & $17(13-23)$ & $22,5(19,5-30)$ & $28(23-33,2)$ & 0,001 \\
TAP (seg)* & $13,8(12,2-16,1)$ & $18,7(15,2-31,5)$ & $15(13,1-19,9)$ & 0,001 \\
TAP (\%)** & $69,3 \pm 21,5$ & $44,5 \pm 22,3$ & $59 \pm 24$ & 0,001 \\
INR* & $1,25(1,12-1,49)$ & $1,72(1,44-2,7)$ & $1,39(1,18-1,84)$ & 0,001 \\
Lactato* & $1,4(1,0-2,0)$ & $2,45(1,75-4,6)$ & $2,2(1,6-4,0)$ & $>0,05$ \\
BE** & $-1,5 \pm 7,0$ & $-6,0 \pm 7,5$ & $-6,4 \pm 9,1$ & 0,001 \\
\hline
\end{tabular}

ANOVA (One way analysis of variance)

* Mediana (25\%-75\%)

**Dados expressos em média \pm DP 
No intuito de identificar um marcador independente para mortalidade precoce, foi realizado uma análise de regressão linear múltipla comparando as variáveis INR, Lactato, $\mathrm{BE}$, idade e APACHE II entre pacientes que foram a óbito em menos de 48 horas e os que sobreviveram por mais de 48 horas. Identificamos o INR como variável independente preditora de evolução para óbito precoce (Tabela 2).

Tabela 2. Regressão Linear Múltipla usando como variável dependente óbito menor que 48 horas.

\begin{tabular}{lcc}
\hline & Escore t & $P$ \\
Idade & 0,190 & 0,849 \\
APACHE II & $-0,395$ & 0,693 \\
INR & 3,705 & $<0,001$ \\
Lactato & $-0,846$ & 0,399 \\
\hline
\end{tabular}

R2 ajustado: 0, 0712

Erro padrão: 0,168

Alfa: 0,050 - 0,968

Dentre os pacientes que sobreviveram por mais de 48 horas, realizamos uma análise semelhante, sendo - APACHE II a variável independente preditora de evolução para óbito tardio (Tabela 3).

Tabela 3. Regressão Linear Múltipla avaliando evolução a óbito dentre os pacientes que sobreviveram às primeiras 48 horas de admissão à UTI.

\begin{tabular}{lll}
\hline & Escore $t$ & $P$ \\
Idade & 1,844 & 0,068 \\
APACHE II & 3,300 & 0,001 \\
INR & 0,645 & 0,520 \\
Lactato & 1,068 & 0,288 \\
BE & $-1,208$ & 0,229 \\
\hline
\end{tabular}

R2 ajustado: 0, 235

Erro padrão: 0, 438

Alfa: 0,050 - 1,000

Realizando uma análise da curva da Característica Operativa do Receptor (ROC) quanto à sensibilidade e especificidade do INR, a área sob a curva encontrada foi de 0,7547 , com um intervalo de confiança de 0,67 a 0 , 83 e valor de $p<0,0001$. O valor de INR que apresentou maior relação entre sensibilidade e especificidade foi de 1, 475 (Figura 1).

\section{DISCUSSÃO}

A incidência de Sepse vem aumentando nos últimos anos, permanecendo como uma das principais causas de óbito dentre os pacientes admitidos à UTI. ${ }^{10}, 11,12$ A despeito da melhoria nas técnicas de monitorização e suporte, a mortalidade dos pacientes que evoluem com sepse grave e choque séptico permanece elevada. No Brasil, o estudo BASES (Brazilian Sepsis Epidemiological Study) mostrou que a mortalidade de pacientes com choque séptico no país chega próximo

\section{Curva ROC}

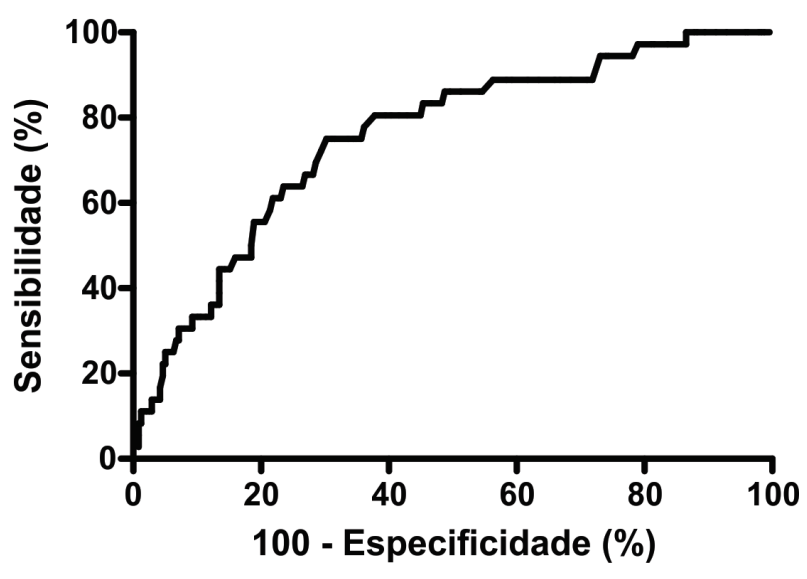

Figura 1

a $60 \%{ }^{13}$ Mesmo com a otimização de fatores da macrocirculação, como pressão arterial e débito cardíaco, os pacientes com sepse podem evoluir com hipóxia tecidual e disfunção metabólica a nível celular. Devido a isso, estudos recentes tem buscado avaliar a microcirculação (definida como vaso sanguíneo de

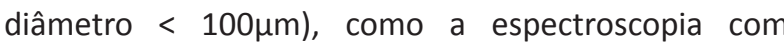
polarização ortogonal (OPS), método que detecta o fluxo circulatório através da mucosa sublingual. ${ }^{14}$ Entretanto, este último método, embora não-invasivo, não se encontra facilmente disponível na UTI e necessita de pessoal especializado.

Os estudos da microcirculação evidenciaram a importância da ativação endotelial, com liberação de citocinas (IL-6, IL-1), fator de necrose tumoral (TNF), formação de espécies reativas do oxigênio e liberação de óxido nítrico, culminando com ativação do sistema de coagulação, com formação de microtrombos, alterações da perfusão regional, hipóxia tecidual, disfunção celular e evolução para disfunção de múltiplos órgãos. ${ }^{15}$ Além disso, as alterações do sistema de coagulação podem por si só podem levar à ativação da cascata inflamatória, num ciclo vicioso perigoso.

Pesquisas recentes avaliando as alterações da coagulação na sepse evidenciaram que a coagulopatia atua como um fator independente de prognóstico, além de estar associada à evolução desfavorável de outros sistemas. As alterações laboratoriais vistas na sepse incluem plaquetopenia, alargamento do TAP e do Tempo de tromboplastina parcial ativada (TTPa), aumento do D-dímero, queda dos níveis de proteína-C e atividade de trombina (AT). Escores prognósticos associando os exames acima já foram usados na avaliação de Coagulação Intravascular Disseminada e Prognóstico na Sepse. ${ }^{7}, 8$

Considerando a importância de identificar precocemente os pacientes que tendem a evoluir com maior gravidade, não somente nos quadros de sepse como também em outras situações clínicas, sempre se 
procura fatores prognósticos mais acurados. Dada a importância das alterações do sistema de coagulação e sua relação com as alterações na microcirculação, procurou-se avaliar a capacidade do mesmo como marcador prognóstico.

A avaliação completa da coagulação envolve uma série de exames (plaquetas, TAP, TTPa, D-Dímero, AT), o que na prática clínica nem sempre se encontra facilmente disponível, além de estar associado a um maior custo. Considerando as características de um marcador ideal como sendo sensível, específico, reprodutível, de fácil acesso e de baixo custo, nenhum dos exames acima satisfaz tais condições. Todavia, como sugerido por Dhainaut e colaboradores, a avaliação do TAP parece apresentar maior peso dentre os exames que avaliam a coagulação. ${ }^{7}$

Além de ser um exame de fácil disponibilidade e de custo aceitável, o TAP ainda apresenta como vantagem o fato de ser expresso como INR, uniformizando dessa forma os valores encontrados. No nosso estudo, encontramos uma associação positiva do INR com a mortalidade precoce ( $<48$ horas), comportando-se como uma variável independente. Estudos abordando as alterações do sistema de coagulação haviam demonstrado associação com mortalidade tardia, ${ }^{15}$ entretanto tais trabalhos utilizaram uma associação de exames na composição de um escore prognóstico próprio e foram realizados somente em pacientes com sepse, enquanto a presente pesquisa utilizou o INR como marcador prognóstico independente do diagnóstico à admissão.

Interessante observar que o valor de INR que apresentou uma melhor sensibilidade e especificidade pela análise da curva $\operatorname{ROC}(1,475)$ é um pouco acima do valor de referência utilizado em nosso laboratório (VR: 1,3 ) e próximo do valor de 1,5 , que já foi sugerido em estudos anteriores como um dos critérios de disfunção hematológica na avaliação de pacientes com sepse grave, alertando para o fato que alterações do INR, que são muitas vezes negligenciadas e tratadas sem maiores ressalvas com reposição de plasma fresco, podem na verdade indicar que o paciente já apresenta alterações orgânicas que confeririam prognóstico desfavorável.

Um ponto desfavorável com relação ao INR é que - mesmo sofre influência de alguns fármacos, principalmente os cumarínicos, devendo este fato ser levado em consideração quando da análise dos valores encontrados. Outro ponto relevante é o que o INR pode estar alargado em pacientes com disfunção hepática, sendo que tanto as alterações da microcirculação como a ativação do sistema de coagulação podem levar a essa disfunção, fato que torna complexa análise da influência deste último fator.

Também se deve considerar o fato do presente estudo ser retrospectivo, o que impede melhor controle das variáveis pesquisadas; ser pontual, avaliando somente os valores da admissão sem observar o padrão de evolução ao longo da internação; ser realizado em centro único, o que impede que se possam extrapolar os resultados para outras populações.

Em contrapartida, o estudo mostrou um resultado inovador, sugerindo o INR como um bom marcador de prognóstico de mortalidade precoce, comportando-se como uma variável independente.

\section{CONCLUSÃO}

O tempo de protrombina se revelou significativamente maior entre os pacientes que faleceram precocemente, sugerindo ser um marcador prognóstico. A sua mensuração na admissão parece ser uma alternativa rápida, segura e de baixo custo para estabelecer o risco de óbito dos pacientes admitidos na UTI. Estudos prospectivos são necessários para confirmar esses resultados.

\section{REFERÊNCIAS}

01. SMITH, I.,et. al. Base excess and lactate as prognostic indicators for patients admitted in intensive care. Intensive Care Med., New York, v.27, n.1, p. 74-83, 2001.

02. RIVERS, E., et al. Early goal-directed thera - py in the treatment of severe sepsis and septic shock. N. Engl. J. Med., Boston, v.345,n.19, p. 1368-1377, 2001.

03. BEZERRA, I.. et. al. Base Deficit at Intensive Care Unit Admission. An Early Mortality Indicator. Rev. Bras. Ter. Int., v.19, n.4, p.434-435, 2007.

04. DAVIS, J.W.; KAUPS, K.L.; PARKS, S.N. Base deficit is superior to $\mathrm{pH}$ in evaluating clearance of acidosis after traumatic shock. J. Trauma., Baltimore, v.44, n. 1, p. 114-118, 1998.

05. LUNDY, D.J.; TRZECIAK, S. Microcirculatory Dysfunction in sepsis. Crit. Care Clin., Philadelphia, v.25, n. 4, p. 721-731, 2009.

06. LEVI, M.; VAN DER POLL, T. Inflammation and coagulation. Crit. Care Med., New York, v. 38, suppl. 2, S26-S34, 2010.

07. DHAINAUT, J.F. et al. Dynamic evolution of coagulopathy in the first day of severe sepsis: Relationship with mortality and organ failure. Crit. Care Med., New York, v. 33, n. 2, p. 341-348, 2005.

08. KINASEWITZ, G.T. et. al. Prognostic value of a simple evolving disseminated intravascular coagulation score in patients with severe sepsis. Crit. Care Med., New York, v. 33, n. 10, p. 2214-2221, 2005.

09. KAMATH, P.S.; KIM, W.R. The International Normalized Ratio of Prothrombin Time in the Model for End-Stage Liver Disease Score: A Reliable Measure. Clin. Liver Dis., Philadelphia, v.13, n.1, p. 63-66, 2009.

10. ANGUS, D.C. et. al. Epidemiology of severe sepsis in the United States: analysis of incidence, outcome, and associated costs of care. Crit. Care Med., New York, v. 29, n.7, p. 1303-10. 2001

11. MARTIN, G.S. et. al. The epidemiology of sepsis in the United States from 1979 through 2000. N. Engl. J. Med., Boston, v.348, p.16, 154654, 2003.

12. BALK, R.A. Severe sepsis and septic shock. Crit. Care Clin., New York, v. 16, n.2, p. 179-91, 2000.

13. SILVA, E. et al. Brazilian Sepsis Epidemiological Study (BASES study). Crit. Care., London, v.8, n.4, p. 251-60, 2004. 
14. RIVERS, E.P.; AHRENS, T. Improving Outcomes for Severe Sepsis and Septic Shock: Tools for Early Identification of At-Risk Patients and Treatment Protocol Implementation. Crit. Care Clin., New York, v.24, suppl. 3, S1-S47, 2008.
15. NIMAH, M.; BRILLI, R.J. Coagulation dysfunction in sepsis and multiple organ system failure. Crit. Care Clin., New York, v. 19, n. 3, p. 441-458, 2003.

Submetido em 01.01.2013

Aceito em 07.06.2013 